\title{
Comparison of Latitude Distribution and Evolution of Even and Odd Sunspot Cycles
}

\author{
Jouni Takalo ${ }^{1}$
}

Received: 14 January 2020 / Accepted: 14 March 2020 / Published online: 30 March 2020

(C) The Author(s) 2020

\begin{abstract}
We study the latitudinal distribution and evolution of sunspot areas of Solar Cycles 12-23 (SC12-23) and sunspot groups of Solar Cycles 8-23 (SC8-23) for even and odd cycles. The Rician distribution is the best-fit function for both even and odd sunspots group latitudinal occurrence. The mean and variance for even northern/southern butterfly wing sunspots are 14.94/14.76 and 58.62/56.08, respectively, and the mean and variance for odd northern/southern wing sunspots are 15.52/15.58 and 61.77/58.00, respectively. Sunspot groups of even cycle wings are thus at somewhat lower latitudes on average than sunspot groups of the odd cycle wings, i.e. about 0.6 degrees for northern hemisphere wings and 0.8 degrees for southern hemisphere wings.

The spatial analysis of sunspot areas between SC12-23 shows that the small sunspots are at lower solar latitudes of the Sun than the large sunspots for both odd and even cycles, and also for both hemispheres.

Temporal evolution of sunspot areas shows a lack of large sunspots after four years (exactly between $4.2-4.5$ years), i.e. about $40 \%$ after the start of the cycle, especially for even cycles. This is related to the Gnevyshev gap and is occurring at the time when the evolution of the average sunspot latitudes crosses about 15 degrees. The gap is, however, clearer for even cycles than odd ones. Gnevyshev gap divides the cycle into two disparate parts: the ascending phase/cycle maximum and the declining phase of the sunspot cycle.
\end{abstract}

Keywords Sun: Sunspot cycle $\cdot$ Sun: sunspot areas $\cdot$ Sun: sunspot groups $\cdot$ Method: distribution analysis $\cdot$ Method: statistical analysis

\section{Introduction}

It has been known almost for two hundred years that the occurrence of sunspots is cyclic, although not strictly periodic. The length of the sunspot cycle (SC) has varied between 9.0 and 13.7 years. The shape of the sunspot cycle has also changed. Waldmeier noticed the

J. Takalo

jouni.j.takalo@oulu.fi

1 Space Physics and Astronomy Research Unit, University of Oulu, POB 3000, 90014, Oulu, Finland 
asymmetry of sunspot cycles, with the ascending phase being typically shorter than the declining phase, and that there is an anti-correlation between cycle amplitude and the length of the ascending phase of the cycle (Waldmeier, 1935, 1939).

Gnevyshev (1967) suggested that the solar cycle is characterized by two periods of activity and these lead to a double peak with the so-called Gnevyshev-gap (GG) in between (Gnevyshev, 1977). Feminella and Storini (1997) studied the long-term behavior of several solar-activity parameters and found that maxima occur at least twice: first, near the end of the rising phase and then in the early years of declining phase. Norton and Gallagher (2010) analyzed the sunspot cycle double peak, and the Gnevyshev gap between them, to determine if the double peak is caused by averaging of the two hemispheres that are out of phase (Temmer et al., 2006). They, however, confirmed previous findings that the Gnevyshev gap is a phenomenon that occurs separately in each hemisphere and is not due to a superposition of sunspot indices from hemispheres slightly out of phase.

Most of the even-odd cycle comparisons have concentrated on the mutual strength of preceding cycles. These are referred as the so-called Gnevyshev-Ohl rule, which is an expression of the general 22-year variation of cycle amplitudes and intensities, according to which even cycles are, on average, about 10-15\% lower than the following odd cycles (Mursula, Usoskin, and Kovaltsov, 2001). There have, however, been some exceptions to this rule, the last one happened between cycle pair SC22-23 (Javaraiah, 2012, 2016).

Another common subject has been the northern-southern asymmetry in solar sunspots and other activity (see some of the recent publications such as: Temmer et al., 2006; Carbonell et al., 2007; Li, Gao, and Zhan, 2009; Chang, 2011; Javaraiah, 2016; Badalyan and Obridko, 2017). Less attention has been paid to statistical distributions of the sunspots, sunspot groups, and areas. There have, however, been some studies about the spatial (latitudinal) distribution of sunspots and sunspot groups using slightly different statistical methods (Li et al., 2003; Ivanov, Miletskii, and Nagovitsyn, 2011; Chang, 2011; Munoz-Jaramillo et al., 2015; Leussu et al., 2016a,b; Mandal, Karak, and Banerjee, 2017).

Li et al. (2003) analyzed the latitudinal distribution of sunspot groups over a solar cycle. They found that although individual sunspot groups of a solar cycle emerge randomly at any middle and low latitude, the whole latitudinal distribution of sunspot groups of the cycle is not stochastic and, in fact, can be represented by a probability density function of the Gamma-distribution type having a maximum probability at about 15.5 degrees of heliographic latitude. Ivanov, Miletskii, and Nagovitsyn (2011) use in their analysis the relative latitude of sunspot groups, i.e. $\phi_{r e l}=\phi-\phi_{o}$, where $\phi_{o}$ is the mean heliographic latitude and $\phi$ is the actual latitude. They show that the latitude distributions of sunspots for a given year can be approximately described by the normal distribution, with its variance being a linear function of the current level of solar activity. Thus, the latitude size of the spotted zone increases with increasing activity. Chang (2011) studied the latitudinal distribution of sunspots between $1874-2009$ by calculating the area-weighted sunspot mean value for each month (he calls it center-of-latitude, COL). He showed that the COL for both northern and southern hemisphere can be fitted with a double Gaussian distribution function.

Munoz-Jaramillo et al. (2015) use 11 different sunspot group, sunspot, and active region databases to characterize the area and flux distributions of photospheric magnetic structures. They plot the fraction of the areas or fluxes per unit area as a function of their area or flux (in millionths of hemisphere, $\mathrm{MH}$ ), and fit different distributions, power-law, log-normal, exponential, and Weibull distributions to the these databases. They found that Weibull (six databases) and log-normal (four databases) are best-fit distributions, but none can explain both the small areas (weak fluxes) and large areas (strong fluxes). They conclude that a composite distribution with Weibull for small areas (weak fluxes) and log-normal for large areas 


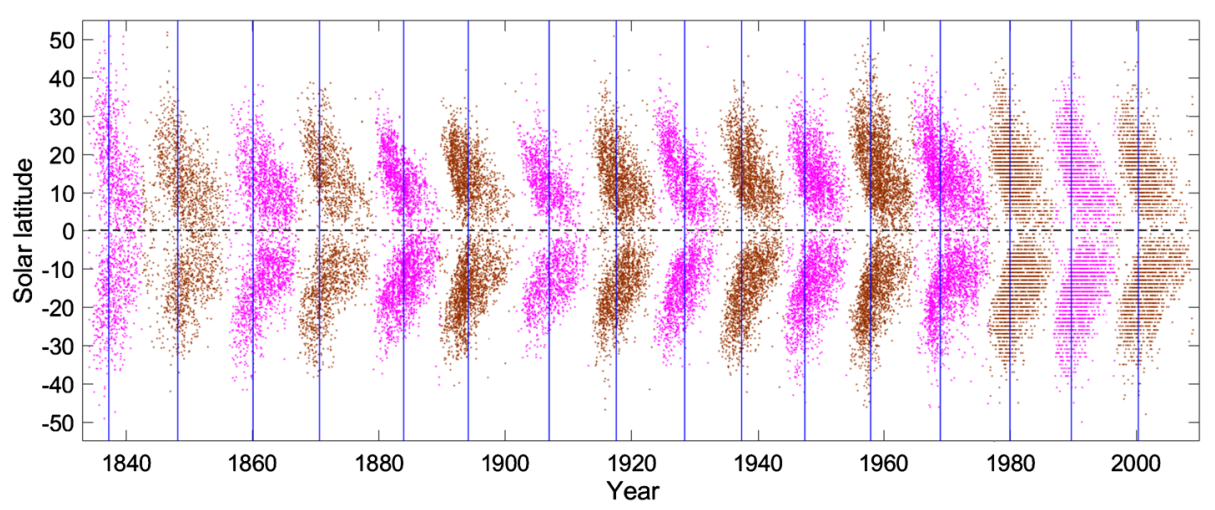

Figure 1 Butterfly pattern of the sunspot groups used in the analysis. The vertical lines correspond to cycle maxima.

(strong fluxes) with transition regions in between can explain best each of their databases. Leussu et al. (2016a) studied especially the latitude evolution and the timing of the sunspot groups in butterfly wings by characterizing three different categories: the latitude at which the first sunspot groups appear, the maximum latitude of sunspot-group occurrence in each wing, and the latitude at which the last sunspot group appears. They derived several statistical measures based on these variables. Mandal, Karak, and Banerjee (2017) found that the latitude distribution of sunspots does not statistically follow a Gaussian distribution. Furthermore, they found that the distribution parameters, the central latitude, width and height show some hemispheric asymmetries and significant positive correlations with the cycle strengths.

In this study we analyze the spatial distribution and evolution of sunspot areas of Solar Cycles (SCs) SC12-23 and sunspot groups of SC8-23 for even and odd cycles, and find the best-fit probability density functions (PDF) for these distributions. This article is organized as follows. Section 2 presents the data and methods used. In Section 3 we study distributions and PDF fits of sunspot groups for even and odd solar cycles. In Section 4 we discuss the latitudinal distribution of sunspot area sizes and migration of the sunspots over the solar cycle. We give our conclusions in Section 5.

\section{Data and Methods}

\subsection{Sunspot Group Data}

In the distribution analysis we use the data set of sunspot groups in southern and northern butterfly wings for cycles SC8-23 by Leussu et al. (Leussu et al., 2016b). This data includes the time and latitude for sunspot groups for cycles SC8-23 and is seen as a butterfly pattern in Figure 1. Note that the cycles SC21-23 have only an integer degree resolution in latitudes.

\subsection{Sunspot Area Sizes}

In the aerial sunspot analysis we use the database of the Royal Observatory, Greenwich USAF/NOAA Sunspot Data for the years 1874-2016 (https://solarscience.msfc.nasa.gov/ greenwch.shtml). This database contains, among others, time, latitude, and area size (in millionths of solar hemisphere, $\mathrm{MH}$ ) for individual sunspots for cycles SC12-23. 


\subsection{Distributions}

\subsubsection{Rayleigh Distribution}

The traditional definition of a normal distribution probability density function (PDF) is

$$
f\left(x ; \mu, \sigma^{2}\right)=\frac{1}{\sqrt{2 \pi \sigma^{2}}} e^{-\frac{(x-\mu)^{2}}{2 \sigma^{2}}},
$$

where $\mu$ is the mean (or expectation) value and $\sigma^{2}$ is the variance. Let us consider a bivariate distribution $(\mathrm{U}, \mathrm{V})$, where $\mathrm{U}$ and $\mathrm{V}$ are normal, independent distributions with zero mean. Then we have

$$
f_{U}\left(u ; \sigma^{2}\right)=\frac{1}{\sqrt{2 \pi \sigma^{2}}} e^{-\frac{u^{2}}{2 \sigma^{2}}}, f_{V}\left(v ; \sigma^{2}\right)=\frac{1}{\sqrt{2 \pi \sigma^{2}}} e^{-\frac{v^{2}}{2 \sigma^{2}}} .
$$

Let us define $X=\sqrt{U^{2}+V^{2}}$. Because $f_{U}$ and $f_{V}$ are perpendicular, their joint bivariate normal distribution, $\mathrm{X}$, has a cumulative distribution

$$
F_{X}\left(x ; \sigma^{2}\right)=\iint_{D_{x}} f_{U}\left(u ; \sigma^{2}\right) f_{V}\left(v ; \sigma^{2}\right) d A,
$$

where $D_{x}$ is the disc $D_{x}=\left\{\sqrt{u^{2}+v^{2}}<x\right\}$. Integration is easier in polar coordinates, where $d A=r d r d \theta$. Writing the double integral in polar coordinates we get

$$
F_{X}\left(x ; \sigma^{2}\right)=\frac{1}{2 \pi \sigma^{2}} \int_{0}^{2 \pi} \int_{0}^{x} r e^{-\frac{r^{2}}{2 \sigma^{2}}} d r d \theta=\frac{1}{\sigma^{2}} \int_{0}^{x} r e^{-\frac{r^{2}}{2 \sigma^{2}}} d r .
$$

While the probability function is the derivative of the cumulative function, we finally get

$$
f_{X}\left(x ; \sigma^{2}\right)=\frac{d}{d x} F_{X}\left(x ; \sigma^{2}\right)=\frac{x}{\sigma^{2}} e^{-\frac{x^{2}}{2 \sigma^{2}}}, x \geq 0,
$$

which is the Rayleigh PDF.

\subsubsection{Rician Distribution}

The Rice distribution, or Rician distribution, is the probability distribution related to the Rayleigh distribution but with non-zero mean (Rice, 1945). The PDF of the Rician distribution is defined as

$$
f_{Z}(z)=\frac{z}{\sigma^{2}} \exp \left(\frac{-\left(z^{2}+v^{2}\right)}{\sigma^{2}}\right) I_{0}\left(\frac{z v}{\sigma^{2}}\right), r \geq 0,
$$

where $I_{0}$ is the modified Bessel function of the first kind with zeroth order (Rice, 1945; Taluktar and Lawing, 1991). Note that for $v=0$ we get the Rayleigh distribution. Note also that $v$ and $\sigma^{2}$ are not the mean and variance of the Rician distribution, but are related to the bivariate Gaussian distribution $x \sim \mathcal{N}\left(v \cos (\theta), \sigma^{2}\right)$ and $y \sim \mathcal{N}\left(v \sin (\theta), \sigma^{2}\right)$ such that $z=\sqrt{x^{2}+y^{2}}$. The mean and variance of the Rician distribution are defined by

$$
\mu=\sigma \sqrt{\pi / 2} L_{1 / 2}\left(-\frac{v^{2}}{2 \sigma^{2}}\right)
$$


and

$$
v a r=2 \sigma^{2}+v^{2}-\frac{\pi \sigma^{2}}{2} L_{1 / 2}^{2}\left(-\frac{v^{2}}{2 \sigma^{2}}\right),
$$

respectively. Here $L_{1 / 2}(\cdot)$ denotes a Laguerre polynomial, which can be presented as

$$
L_{1 / 2}(\xi)=\exp ^{1 / 2}\left[(1-\xi) I_{0}\left(-\frac{\xi}{2}\right)-x I_{1}\left(-\frac{\xi}{2}\right)\right] .
$$

\subsection{Statistical Methods}

\subsubsection{Negative Log-Likelihood}

The likelihood function $L(\theta)$ is defined as

$$
L(\theta)=\prod_{i=1}^{n} f_{\theta}\left(x_{i}\right)
$$

if variables $x_{i}$ are independent and from the same distribution $f_{\theta}$. The set of parameters $\theta$ of the distribution, which maximizes $L(\theta)$ is called a maximum likelihood estimator (MLE) and is denoted as $\theta_{L}$. It is often easier to maximize the $\log$-likelihood function, $\log L(\theta)$, and since the (natural) logarithmic function is monotonically increasing, the same value maximizes both $L(\theta)$ and $\log L(\theta)$. Because the log-likelihoods here are always negative, we calculate instead the minimum value for negative $\log$-likelihood (NLogL) (Forbes et al., 2011).

\subsubsection{Two-Sample T-Test}

The two-sample T-test for equal mean values is defined as follows. The null hypothesis assumes that the means of the samples are equal, i.e. $\mu_{1}=\mu_{2}$. An alternative hypothesis is that $\mu_{1} \neq \mu_{2}$. The test statistic is calculated as

$$
T=\frac{\mu_{1}-\mu_{2}}{\sqrt{s_{1}^{2} / N_{1}+s_{2}^{2} / N_{2}}},
$$

where $N_{1}$ and $N_{2}$ are the sample sizes, $\mu_{1}$ and $\mu_{1}$ are the sample means, and $s_{1}^{2}$ and $s_{2}^{2}$ are the sample variances. If the sample variances are assumed equal, the formula reduces to

$$
T=\frac{\mu_{1}-\mu_{2}}{s_{p} \sqrt{1 / N_{1}+1 / N_{2}}},
$$

where

$$
s_{p}^{2}=\frac{\left(N_{1}-1\right) s_{1}^{2}+\left(N_{2}-1\right) s_{2}^{2}}{N_{1}+N_{2}-2} .
$$

The rejection limit for two-sided T-test is $|T|>t_{1-\alpha / 2, \nu}$, where $\alpha$ denotes significance level and $v$ degrees of freedom. The values of $t_{1-\alpha / 2, v}$ are published in T-distribution tables (Snedecor and Cochran, 1989; Krishnamoorthy, 2006; Derrick, Deirdre, and White, 2016). Now, if the value of $\mathrm{p}<\alpha=0.05$, the significance is at least $95 \%$, and if $\mathrm{p}<\alpha=0.01$, the significance is at least $99 \%$. 

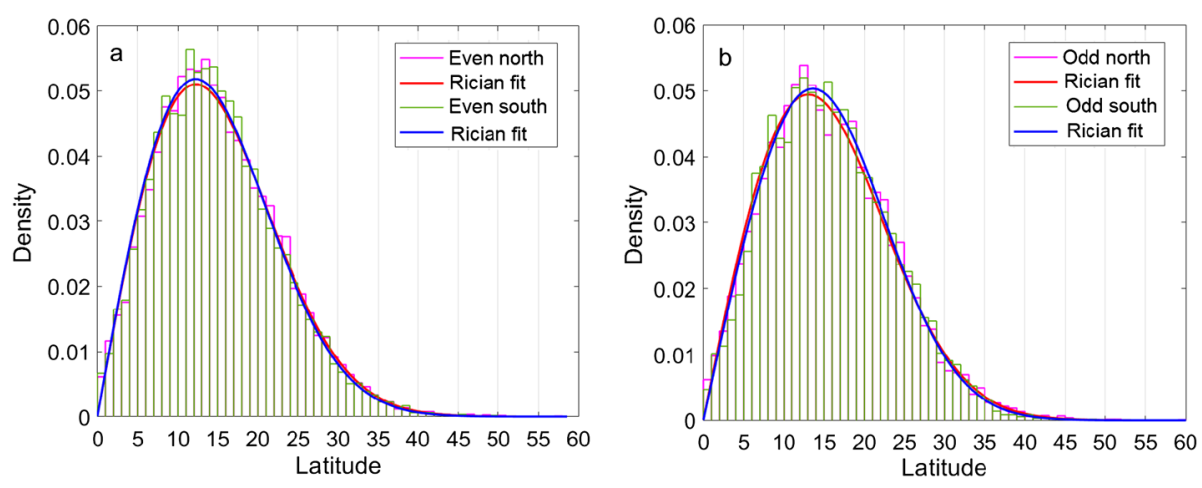

Figure 2 a) The latitudinal probability density of northern and southern (absolute value) hemisphere sunspot groups with their Rician distribution fits for even cycles in the range SC8-23. b) Same as a) but for odd cycles.

\section{Latitudinal Distribution of Sunspot Groups for Even and Odd Cycles}

Figures $2 \mathrm{a}$ and $2 \mathrm{~b}$ show the latitudinal distribution histograms of northern and southern (absolute value) hemisphere sunspot groups with their Rician distribution fits for even and odd cycles SC8-23 in the range, respectively. It is clear that the distributions are not normal because of the cutoff at zero and positive skewness. The NLogL of the Rician distribution fits for even northern/southern wing groups are 40761/40770, while NLogL for, e.g., lognormal distribution fits are 42347/42417. Almost as good fits as Rician are Rayleigh (NLogL $=40766 / 40788)$ and Weibull (40764/40774) distribution fits. The NLogL of Rician distribution fits for odd northern/southern wing sunspots are 46803/44526, while NLogL for lognormal distribution fits are 48770/46374. The Rician distribution parameters for even northern/southern wing sunspot are $v=8.262$ (standard error, $\mathrm{SE}=0.581) / 8.730(\mathrm{SE}=0.443$ ) and $\sigma=10.338(\mathrm{SE}=0.234) / 9.944(\mathrm{SE}=0.196)$ and the Rician distribution parameters for odd northern/southern wing sunspots are $v=9.822(\mathrm{SE}=0.334) / 11.072(\mathrm{SE}=0.208)$ and $\sigma=10.162(\mathrm{SE}=0.167) / 9.43713(\mathrm{SE}=0.119)$. The mean and variance for even northern/southern sunspot groups are 14.94/14.76 and 58.62/56.08, respectively, and the mean and variance for odd northern/southern sunspot groups are 15.52/15.58 and 61.77/58.00, respectively. This shows that the sunspot groups of even cycle wings are on the average at somewhat lower latitudes than sunspot groups of the odd cycle wings, i.e. about 0.6 degrees for the northern hemisphere groups and 0.8 degrees for the southern hemisphere groups. Two-sample T-test gives significance for the difference of the means between even and odd cycles with $\mathrm{p}<10^{-9}$ and $\mathrm{p}<10^{-16}$ for north sunspot groups and south sunspot groups, respectively. This means that significance is much better than $99 \%$ in both cases.

\section{Distribution of Sunspot Areas}

\subsection{Latitudinal Distribution of Sunspot Areas}

Figures $3 \mathrm{a}$ and $3 \mathrm{~b}$ show the density of all sunspots (independent of the area) as histograms and the total areas of the sunspots at each (integer) latitude for even and odd cycles between SC12 - 23, respectively. The distribution and areal strength of the sunspots are quite similar 

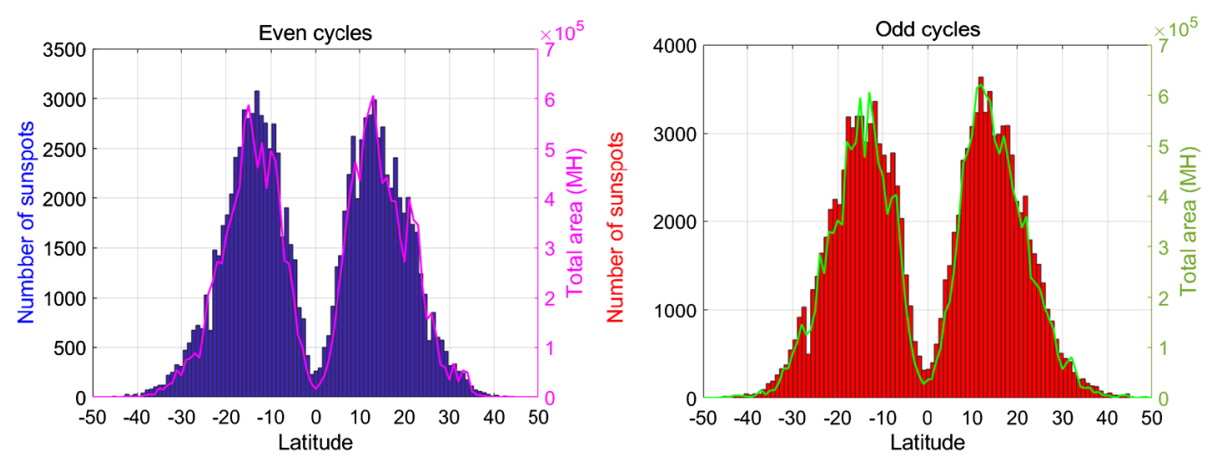

Figure 3 a) The number of all sunspots of even cycles in the range SC12-22 as histograms and their total areas (left vertical axis) for both northern and southern solar hemisphere. b) Same as a) but for all sunspots of odd cycles in the range $\mathrm{SC} 13-23$.

but do not coincide in the low latitudes. It seems that there are smaller sunspots at low latitudes of the Sun for both odd and even cycles, and also for both hemispheres. Note that the histograms are somewhat narrower for even cycles than for odd cycles in both hemispheres. The averages latitudes are 15.02 and -14.79 for the northern and southern hemisphere for even cycles, respectively, and 15.51 and -15.55 for the northern and southern hemisphere for odd cycles, respectively. Again the average latitudes are about 0.5 and 0.8 degrees lower for even cycles than odd cycles. The average sizes of sunspots are 165.5 and $159.1 \mathrm{MH}$ for even and odd cycles, respectively.

Figures $4 \mathrm{a}$ and $4 \mathrm{c}$ show the total areas of large sunspots (area $\geq 500 \mathrm{MH}$ ), medium size sunspots $(100 \leq$ area $<500 \mathrm{MH})$, and small sunspots (area $<100 \mathrm{MH}$ ) and number as histograms at each latitude in Figures $4 \mathrm{~b}$ and $4 \mathrm{~d}$ for even and odd cycles, respectively. It seems that the largest sunspots are located at latitudes between absolute values 10-25 degrees for both hemispheres and both even and odd cycles. There also seems to be some (quasi) periodicity in the locations of the large sunspots, especially for odd cycles. Note that for both hemispheres for even cycles and southern hemisphere for odd cycles there is a gap in the histogram of large sunspots around latitude \pm 15 degrees. The small sunspots are most abundant and spread from zero to 40 degrees but their total area is much smaller than the total areas of medium sized and large sunspots. The total areas of all sunspots are $1.78 \times 10^{7}$ and $2.01 \times 10^{7} \mathrm{MH}$ for even and odd cycles of the range SC12-23, respectively.

\subsection{Temporal Distribution and Migration of Sunspots}

Figures $5 \mathrm{a}$ and $5 \mathrm{~b}$ show the density of very large sunspot distributions (areas equal or over $1000 \mathrm{MH}$ ) for even and odd cycles between SC12-23, respectively. The time-resolution of the figures is 0.1 year. In the same figures we show the average and median latitudes of all sunspots (right-axis) as a function of time for the whole period SC12-23 (absolute value is taken for southern hemisphere sunspots). The most conspicuous feature for the even cycles is the lack of large sunspots for four years (exactly between 4.2-4.5 years) after the start of the cycle. Two-sided T-test shows that this gap has significantly different mean value (at level 99\%) with $\mathrm{p}=0.0022$ than the one-year period before and after the gap. Furthermore, the gap seems to be located at about the time when the evolution of the average sunspot latitude crosses about 15 degrees solar latitude. We believe that this is related to the Gnevyshev-gap (Gnevyshev, 1967, 1977; Storini et al., 2003; Ahluwalia and Kamide, 2004; 

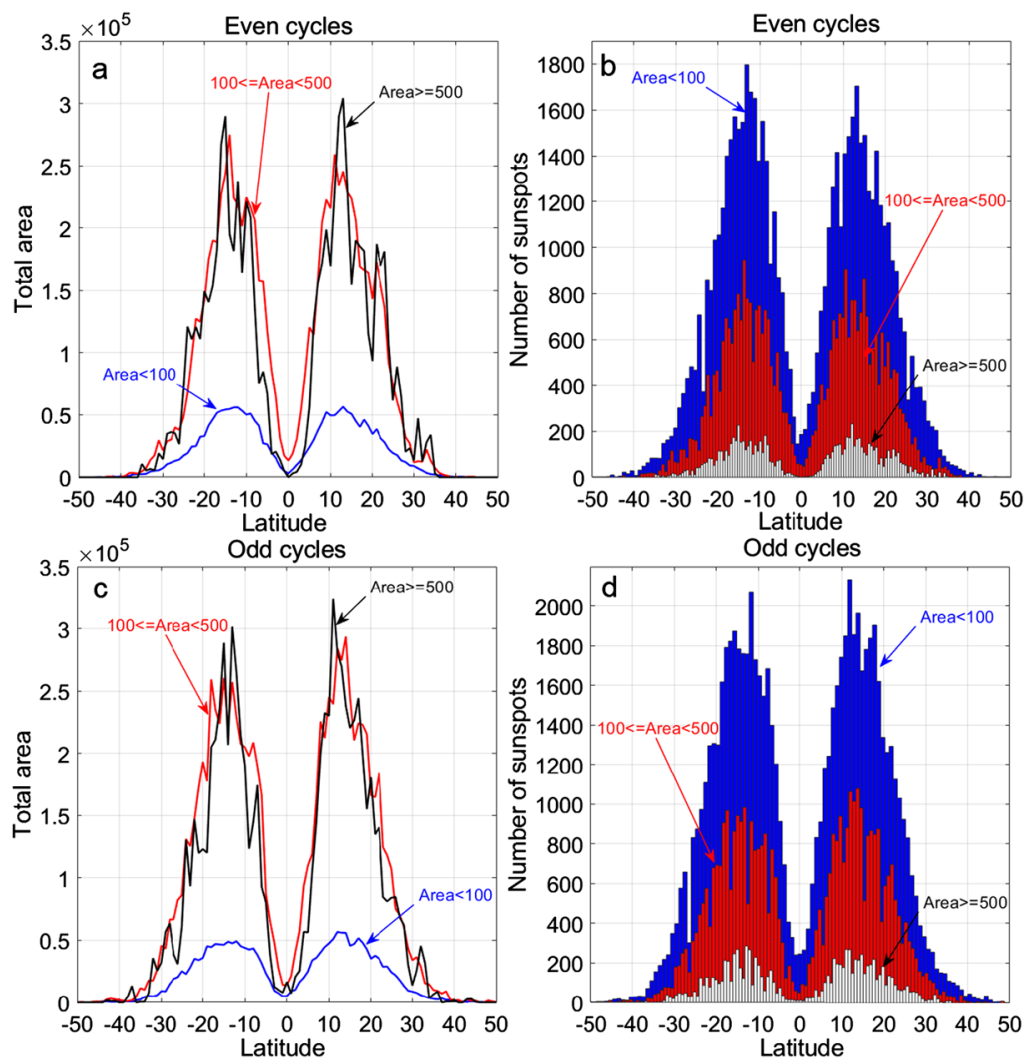

Figure 4 a) Total areas of large sunspots (area $\geq 500 \mathrm{MH}$ ), medium sunspots $(100 \leq$ area $<500 \mathrm{MH}$ ), and small sunspots (area $<100 \mathrm{MH}$ ) of even cycles in the range SC12-22 and b) the number of each size as histograms for both northern and southern solar hemisphere for even cycles. c) Same as a) but for sunspots of odd cycles in the range SC13-23 and d) same as c) but for odd cycles.

Bazilevskaya, Makhmutov, and Sladkova, 2006; Norton and Gallagher, 2010; Du, 2015; Takalo and Mursula, 2018). The distribution of odd cycles has only a small decrease in the distribution at $4.2-4.3$ years, but it is insignificant according to two-sided T-test analysis $(\mathrm{p}=0.40)$.

\section{Conclusions}

We have analyzed the spatial and temporal distribution of sunspot groups for even and odd cycles in the range SC8-23. The Rician distribution is the best-fit function for both even and odd sunspot-group latitudinal occurrence. The mean and variance for northern/southern wing sunspots are 14.94/14.76 and 58.62/56.08 for even cycles, respectively, and $15.52 / 15.58$ and $61.77 / 58.00$ for odd cycles, respectively. This shows that the sunspot groups of even cycle wings are on the average at somewhat lower latitudes than sunspot groups of the odd cycle wings, i.e. about 0.6 degrees for northern hemisphere wings and 0.8 degrees for southern hemisphere wings. Furthermore, the distributions for even cycles are slightly narrower than for odd cycles. The skewnesses for all (absolute value) even/odd 

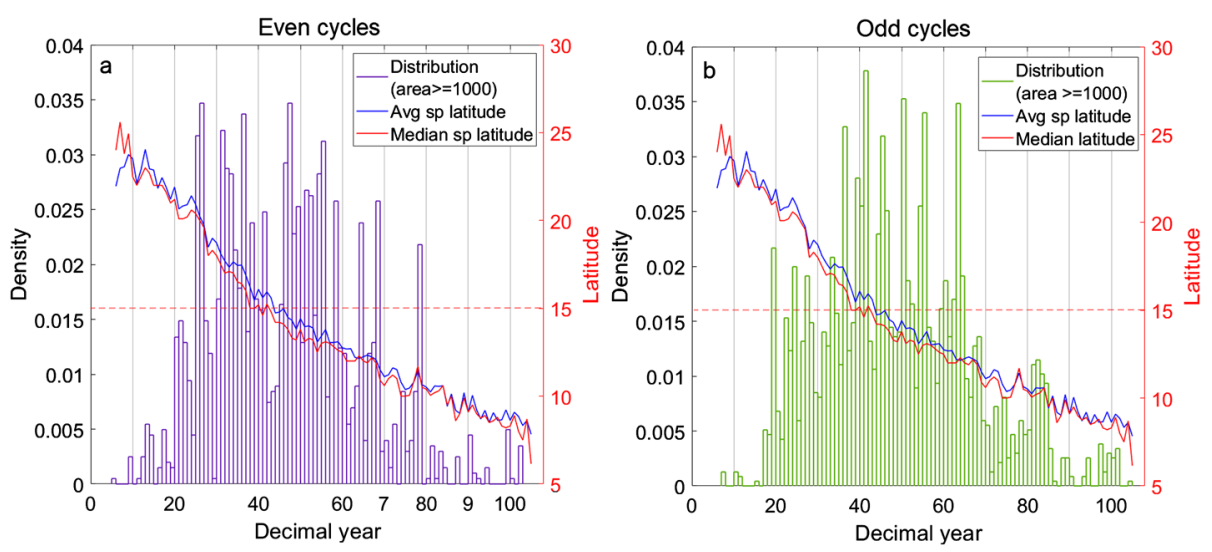

Figure 5 a) The density of the large sunspot distribution for even cycles in the range SC12-22 as a function of decimal year (unit $=0.1$ year). The blue and red curves show the mean and median latitude of these sunspots as a function of time (the latitude axis on the right). b) Same as a) but for large sunspots of odd cycles in the range $\mathrm{SC} 13-23$.

cycle distributions are $0.589 / 0.500$ and the kurtoses $3.303 / 3.147$, which verify the abovementioned results.

The spatial analysis of sunspot areas between SC12 - 23 shows that the small sunspots are at lower latitudes of the Sun than the large sunspots for both odd and even cycles, and also for both hemispheres. The total area of the sunspots is more symmetric for the hemispheres of the odd cycles than for those of the even cycles. The large area sunspots ( $\geq 1000 \mathrm{MH})$ occur mainly between 10 and 25 degrees at both northern and southern hemisphere and for both even and odd cycles. For even cycles there is a gap at $15(-15)$ degrees northern (southern) hemisphere latitudes in the occurrence of large sunspots, but for odd cycles the amount of large sunspots abruptly decreases after $15(-15)$ degrees northern (southern) latitude.

The temporal evolution of sunspot areas shows a lack of large sunspots for four years (exactly between $4.2-4.5$ years), i.e., about $40 \%$ after the start of the cycle. This is related to the Gnevyshev gap and is consistent with the earlier result by Takalo and Mursula (2018). The significance level of this gap for even cycles is at least $95 \%$ for all sunspots and still higher (99\%) for large sunspots. Furthermore, the average of the sunspot areas in this interval for even cycles is $152 \mathrm{MH}$, while in the surrounding interval (year before and year after) it is $188 \mathrm{MH}$. We have also shown that this gap is temporally located at the same time as the average latitude of the migration of the sunspots crosses $15(-15)$ degrees of northern (southern) heliographic latitude. For odd cycles the gap is narrower (4.2-4.3 years) and it is insignificant according to two-sample T-test for the means.

Acknowledgements Open access funding provided by University of Oulu including Oulu University Hospital. We acknowledge the financial support by the Academy of Finland to the ReSoLVE Centre of Excellence (project no. 272157). The author thanks prof. K. Mursula for useful discussions. The sunspot-group data are downloaded from VizieR database (https://vizier.u-strasbg.fr/viz-bin/VizieR?-source=J/A+A/599/A131\&-to=3), and the sunspot area data from RGO-USAF/ NOAA (https://solarscience.msfc.nasa.gov/greenwch.shtml). The dates of cycle maxima were obtained from the National Geophysical Data Center (NGDC), Boulder, Colorado, USA (ftp.ngdc.noaa.gov).

Disclosure of Potential Conflicts of Interest The author declares that there are no conflicts of interest. 
Publisher's Note Springer Nature remains neutral with regard to jurisdictional claims in published maps and institutional affiliations.

Open Access This article is licensed under a Creative Commons Attribution 4.0 International License, which permits use, sharing, adaptation, distribution and reproduction in any medium or format, as long as you give appropriate credit to the original author(s) and the source, provide a link to the Creative Commons licence, and indicate if changes were made. The images or other third party material in this article are included in the article's Creative Commons licence, unless indicated otherwise in a credit line to the material. If material is not included in the article's Creative Commons licence and your intended use is not permitted by statutory regulation or exceeds the permitted use, you will need to obtain permission directly from the copyright holder. To view a copy of this licence, visit http://creativecommons.org/licenses/by/4.0/.

\section{References}

Ahluwalia, H.S., Kamide, Y.: 2004, Gnevyshev gap, Forbush decrease, ICME/SSC, and solar wind. In: Paillé, J.-P. (ed.) 35th COSPAR Scientific Assembly, 470. ADS.

Badalyan, O.G., Obridko, V.N.: 2017, North-South asymmetry of solar activity as a superposition of two realizations - the sign and absolute value. Astron. Astrophys. 603, A109. DOI.

Bazilevskaya, G.A., Makhmutov, V.S., Sladkova, A.I.: 2006, Gnevyshev gap effects in solar energetic particle activity. Adv. Space Res. 38, 484. DOI.

Carbonell, M., Terradas, J., Oliver, R., Ballester, J.L.: 2007, The statistical significance of the North-South asymmetry of solar activity revisited. Astron. Astrophys. 476(2), 951. DOI. ADS.

Chang, H.-Y.: 2011, Bimodal distribution of area-weighted latitude of sunspots and solar North-South asymmetry. New Astron. 17(3), 247. DOI. ADS.

Derrick, B., Deirdre, T., White, P.: 2016, Why Welch's test is type I error robust. Quant. Methods Psychol. 12(1), 30. DOI. http://eprints.uwe.ac.uk/27232.

Du, Z.L.: 2015, Bimodal structure of the solar cycle. Astrophys. J. 804, 15. DOI. ADS.

Feminella, F., Storini, M.: 1997, Large-scale dynamical phenomena during solar activity cycles. Astron. Astrophys. 322, 311. ADS.

Forbes, C., Evans, N., Hastings, N., Peacock, B.: 2011, Statistical Distributions 4, Wiley, Hoboken, 47.

Gnevyshev, M.N.: 1967, On the 11-years cycle of solar activity. Solar Phys. 1, 107. DOI. ADS.

Gnevyshev, M.N.: 1977, Essential features of the 11-year solar cycle. Solar Phys. 51, 175. DOI. ADS.

Ivanov, V.G., Miletskii, E.V., Nagovitsyn, Y.A.: 2011, Form of the latitude distribution of sunspot activity. Astron. Rep. 55(10), 911. DOI. ADS.

Javaraiah, J.: 2012, The G-O rule and Waldmeier effect in the variations of the numbers of large and small sunspot groups. Solar Phys. 281, 827. DOI.

Javaraiah, J.: 2016, North-South asymmetry in small and large sunspot group activity and violation of evenodd solar cycle rule. Astrophys. Space Sci. 361, 208. DOI. ADS.

Krishnamoorthy, K.: 2006, Handbook of Statistical Distributions with Applications, Chapman \& Hall/CRC Press/Taylor \& Francis, London, Boca Raton, 128. 33487-2742, ISBN 1-58488-635-8.

Leussu, R., Usoskin, I.G., Arlt, R., Mursula, K.: 2016a, Properties of sunspot cycles and hemispheric wings since the 19th century. Astron. Astrophys. 592, A160. DOI. ADS.

Leussu, R., Usoskin, I.G., Senthamizh Pavai, V., Diercke, A., Arlt, R., Mursula, K.: 2016b, VizieR Online Data Catalog: Butterfly diagram wings (Leussu+, 2017). VizieR Online Data Catalog 359. https://vizier. u-strasbg.fr/viz-bin/VizieR?-source=J/a+a/599/A131\&-to=3.

Li, K.J., Gao, P.X., Zhan, L.S.: 2009, The long-term behavior of the North South asymmetry of sunspot activity. Solar Phys. 254(1), 145. DOI. ADS.

Li, K.J., Wang, J.X., Zhan, L.S., Yun, H.S., Liang, H.F., Zhao, H.J., Gu, X.M.: 2003, On the latitudinal distribution of sunspot groups over a solar cycle. Solar Phys. 215(1), 99. DOI. ADS.

Mandal, S., Karak, B.B., Banerjee, D.: 2017, Latitude distribution of sunspots: analysis using sunspot data and a dynamo model. Astrophys. J. 851(1), 70. DOI.

Munoz-Jaramillo, A., Senkpeil, R.R., Windmueller, J.C., Amouzou, E.C., Longcope, D.W., Tlatov, A.G., Nagovitsyn, Y.A., Pevtsov, A.A., Chapman, G.A., Cookson, A.M., Yeates, A.R., Watson, F.T., Balmaceda, L.A., DeLuca, E.E., Martens, P.: 2015, Small-scale and global dynamos and the area and flux distributions of active regions, sunspot groups, and sunspots: a multi-database study. Astrophys. J. 800(1), 48. DOI.

Mursula, K., Usoskin, I.G., Kovaltsov, G.A.: 2001, Persistent 22-year cycle in sunspot activity: evidence for a relic solar magnetic field. Solar Phys. 198(1), 51. DOI. ADS. 
Norton, A.A., Gallagher, J.C.: 2010, Solar-cycle characteristics examined in separate hemispheres: phase, Gnevyshev gap, and length of minimum. Solar Phys. 261, 193. DOI. ADS.

Rice, S.O.: 1945, Mathematical analysis of random noise. Bell Syst. Tech. J. 24, 46.

Snedecor, G.W., Cochran, W.G.: 1989, Statistical Methods, 8th edn. Iowa State University Press and WileyBlackwell, Ames, Oxford, 64.

Storini, M., Bazilevskaya, G.A., Fluckiger, E.O., Krainev, M.B., Makhmutov, V.S., Sladkova, A.I.: 2003, The GNEVYSHEV gap: a review for space weather. Adv. Space Res. 31, 895. DOI.

Takalo, J., Mursula, K.: 2018, Principal component analysis of sunspot cycle shape. Astron. Astrophys. 620, A100. DOI.

Taluktar, K.K., Lawing, W.D.: 1991, Estimation of the parameters of the Rice distribution. J. Acoust. Soc. Am. 89(3), 1191. DOI.

Temmer, M., Rybák, J., Bendík, P., Veronig, A., Vogler, F., Otruba, W., Pötzi, W., Hanslmeier, A.: 2006, Hemispheric sunspot numbers $\left\{\mathrm{R}_{n}\right\}$ and $\left\{\mathrm{R}_{S}\right\}$ from 1945-2004: catalogue and N-S asymmetry analysis for solar cycles $18-23$. Astron. Astrophys. 447(2), 735. DOI. ADS.

Waldmeier, M.: 1935, Neue Eigenschaften der Sonnenfleckenkurve. Astron. Mitt. Zür. 14, 105.

Waldmeier, M.: 1939, Die Zonenwanderung der Sonnenflecken. Astron. Mitt. Zür. 14, 470. ADS. 